\title{
Congenital Myasthenia Gravis: A Case Report
}

\author{
Bhramara Madduri ${ }^{1 *}$, Seemantini Ayachit ${ }^{2}$, Milena Dimic ${ }^{3}$, Praveen Kumar ${ }^{4}$ and Neeta Raju ${ }^{5}$ \\ ${ }^{1}$ Department of Pediatrics and Neonatology, Royal Bahrain hospital, Bahrain \\ ${ }^{2}$ Fellowship Pediatric Ophthalmology, Royal Bahrain hospital, Bahrain \\ ${ }^{3}$ Department of Pediatric Neurologist, Royal Bahrain Hospital, Bahrain \\ ${ }^{4}$ Fellowship Pediatric Neurology, Royal Bahrain hospital, Bahrain \\ ${ }^{5}$ Department of Pediatrics, Royal Bahrain hospital, Bahrain
}

*Corresponding author: Bhramara Madduri, Department of Pediatrics and

Neonatology, Royal Bahrain hospital, Bahrain.

Received Date: November 01, 2020

Published Date: November 16, 2020

\section{Case Report}

Congenital myasthenia gravis (CMS) is not very uncommon but could be easily missed. Timely identification and management can improve quality of life. Here we present a case of congenital myasthenia gravis, confirmed by genetic studies, detected at 2 years of age.

A 2 year 1 month old girl child was brought by her parents to the pediatric outpatient clinic for the first time (she was seen in a different facility earlier) with fever, cough and wheezing suggestive of acute bronchitis. She was born $2.7 \mathrm{~kg}$, at 37 weeks by elective LSCS to third degree consanguineous parents. Antenatally mother noticed decreased fetal movements from the beginning. Baby had a weak and feeble cry after birth but had normal APGAR scores. She was admitted in hospital twice, once at 2 months of age for aspiration pneumonia and second time later in infancy for pneumonia.

Parents noticed that she would be energetic soon after waking up in the morning, and her eyes seemed wide open as compared to the evening when her eyes seemed droopy and she was low in her activity. Even a short nap would get her back to normal. She was unable to climb stairs or get down stairs, according to the parents.

On examination, seen at 6:45 pm, she was a thin, frail, pale looking girl, shy, with puffy eye lids, mask like face, and droopy eyelids.
There was severe bilateral ptosis, compensatory head posture, frontalis over action and her muscles were flabby, with normal tendon reflexes. Tripod test was positive indicating proximal muscle weakness. She rarely smiled.

She was diagnosed and treated for bronchitis; and suspect of having Myasthenia gravis with a differential diagnosis of Congenital myopathy, underwent ophthalmological and pediatric neurological evaluation.

Ophthalmologic examination revealed that visual acuity could not be recorded as the child was noncooperative and irritable. Light reflexes were central, and the child was able to follow near and distant objects well, but the ocular movements were slow and partial ophthalmoplegia was observed. There was poor eye contact. Ptosis improved with short periods of rest as noticed in the ophthalmology clinic. The morning photographs of the patient showed marked improvement in ptosis and expression. Anterior segment examination was unremarkable, and fundus was within normal limits.

Neurological examination showed a generalized developmental delay, feeble voice and cry, hypotonia, and ptosis. The child was unable to climb the steps and Tripod test was positive. Her Icepack test was also positive. 
Anticholinesterase antibodies were negative. CT chest was done to rule out thymoma, which was within normal limits. MRI brain was within normal limits. A 2 D echocardiography revealed no abnormality.

Genetic test reports of the child identified congenital myasthenic syndrome- $4 c$, of autosomal recessive inheritance. She had high lactate levels of $3.1 \mathrm{mmol} / \mathrm{L}$.

She was started on pyridostigmine tablets resulting in improvement in all signs and symptoms within a few days.

The earliest report of Congenital Myasthenia Gravis was by Rothbart in 1937 while the term "congenital myasthenia" was coined by Bowman to describe an infant whose symptoms persisted into childhood but had normal parents. Severity ranges from mild, phasic weakness, to disabling, permanent muscle weakness, respiratory insufficiency, and early death [1]. Earlier, CMS was diagnosed by combined clinical, in vitro electrophysiological, and structural studies. With the advent of Sanger sequencing and whole exam sequencing in the recent years, around 20 CMS disease genes have been identified so far. The commoner genes associated with CMS are RAPSN, CHRNE, DOK7 and GFPT1 [1,2]. Clinical phenotypes of congenital myasthenic syndromes and primary mitochondrial disorders share significant overlap in their clinical presentations, leading to challenges in making the correct diagnosis. Next generation sequencing is transforming molecular diagnosis of inherited neuromuscular disorders by identifying novel disease genes and by identifying previously known genes in undiagnosed patient [3]. In our case, a homozygous single base pair duplication was detected in exon 2 of the CHRNE gene (chr17:4805975dupC; Depth: 45x) that results in a frameshift and premature truncation of the protein 3 amino acids downstream to codon 44. A heterozygous missense variation in exon 14 of the MYH2 gene (chr17:10442605G>A; Depth: 130x) that results in the amino acid substitution of Cysteine for Arginine at codon 445 (p.Arg445Cys; ENST00000245503.5) was also detected. Though this genetic mutation is implicated in Proximal myopathy and ophthalmoplegia, its significance is not known in relation to congenital myasthenia gravis [4].

In juvenile myasthenia, clinical features mainly comprise of ptosis and some degree of extra ocular muscle weakness as the earliest and most constant symptoms [5].

Prognosis of CMS is difficult to predict. Those that occur beyond neonatal period, with early diagnosis and appropriate treatment, have the most favorable outcome.

\section{Acknowledgement}

None.

\section{Conflict of Interest}

No conflict of interest.

\section{References}

1. Finsterer J (2019) Congenital Myasthenic Syndromes. Orphanet J Rare Diseases 14(1): 57.

2. Natera-de Benito D, Bestué M, Vilchez JJ, Evangelista T, Töpf A, et al. (2016) Long-term follow-up in patients with congenital myasthenic syndrome due to RAPSN mutations. Neuromuscul Disord 26(2): 153159.

3. Yiran Guo, Minal Menezes, Manoj Menezes, Jinlong Liang, Dong Li, et al. (2015) Delayed diagnosis of congenital myasthenia due to associated mitochondrial enzyme defect. Neuromuscul Disord 25(3): 257-261.

4. Homa Tajsharghi, Simon Hammans, Christopher Lindberg, Alexander Lossos, Nigel F Clarke, et al. (2014) Recessive myosin myopathy with external ophthalmoplegia associated with $\mathrm{MYH}_{2}$ mutations. Eur J Hum Genet 22(6): 801-808.

5. Tsao CY (2016) Effective Treatment with Albuterol in DOK $\mathrm{K}_{7}$ Congenital Myasthenic Syndrome in Children. Pediatr Neurol 54: 85-87. 